\title{
T cells have a pivotal role in autoimmune pancreatitis in an experimental mouse model
}

Experimental studies in mice have indicated that $\mathrm{T}$ cells have a major role in inducing autoimmune pancreatitis (AIP), and that ciclosporin and sirolimus (also known as rapamycin) could be alternatives to standard steroid treatment.

AIP is a form of chronic pancreatitis that usually responds to steroids, but relapse can occur and little is known about the effectiveness of alternative immunosuppressants. "Analogous to the treatment of IBD, a steroid-sparing therapy is desirable," says author Julia Mayerle.

Mayerle and colleagues used a mouse model that mimics human type I AIP (MRL/Mp mice). Using this model, they investigated the role of T cells in the development of AIP by blocking CTLA4 (a potent attenuator of T-cell response) and the therapeutic potential of sirolimus, azathioprine and ciclosporin.

Blockade of CTLA4 in MRL/Mp mice led to increased severity of AIP with pronounced organ destruction and inflammatory cell infiltration. The composition of T-cell subsets in these AIP mice was also altered; T-regulatory $\left(\mathrm{T}_{\mathrm{REG}}\right)$ cells were suppressed, whereas the effector T-cell response increased.

Treatment with sirolimus and ciclosporin markedly reduced AIP severity, but by different mechanisms; ciclosporin decreased $\mathrm{CD} 69^{+} \mathrm{CD} 4^{+}$effector $\mathrm{T}$ cells, whereas sirolimus increased $\mathrm{T}_{\mathrm{REG}}$ cells, which in turn suppressed effector T-cell responses. Azathioprine did not reduce AIP severity, with minimal effect on T-cell populations.

The authors propose that ciclosporin and sirolimus are promising therapeutic candidates in humans with AIP and should be tested in clinical trials.

\section{Katrina Ray}

Original article Schwaiger, T. et al. Autoimmune pancreatitis in MRL/Mp mice is a T cell-mediated disease responsive to cyclosporine $A$ and rapamycin treatment. Gut doi:10.1136/gutjnl-2012-303635

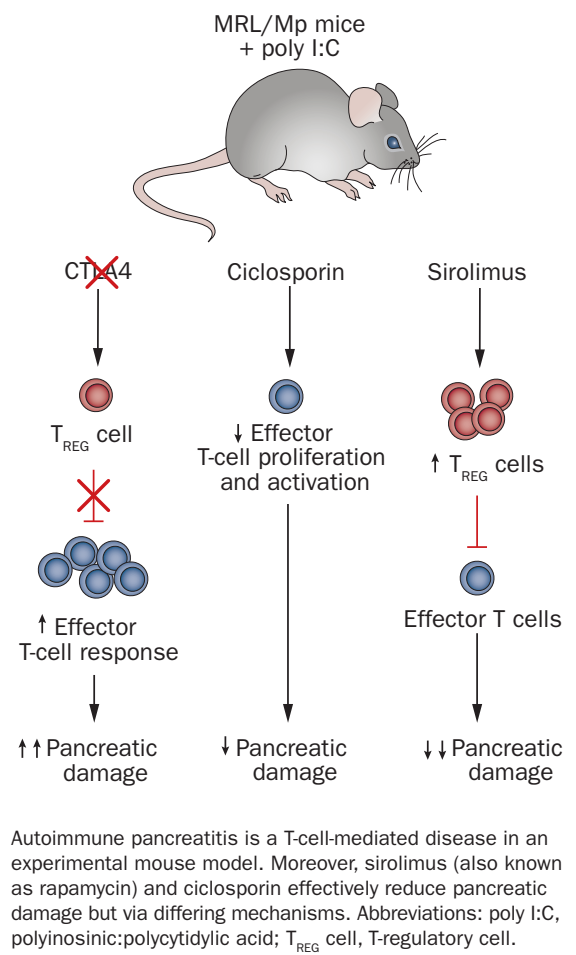

